Homology, Homotopy and Applications, vol.20(1), 2018, pp.251-257

\title{
TENSORING WITH THE FROBENIUS ENDOMORPHISM
}

\author{
OLGUR CELIKBAS, ARASH SADEGHI AND YONGWEI YAO
}

\author{
(communicated by Winfried Bruns)
}

\begin{abstract}
Let $R$ be a commutative Noetherian Cohen-Macaulay local ring that has positive dimension and prime characteristic. Li proved that the tensor product of a finitely generated non-free $R$-module $M$ with the Frobenius endomorphism $\varphi^{n} R$ is not maximal CohenMacaulay provided that $M$ has rank and $n \gg 0$. We replace the rank hypothesis with the weaker assumption that $M$ is locally free on the minimal prime ideals of $R$. As a consequence, we obtain, if $R$ is a one-dimensional non-regular complete reduced local ring that has a perfect residue field and prime characteristic, then ${ }^{\varphi^{n}} R \otimes_{R} \varphi^{n} R$ has torsion for all $n \gg 0$. This property of the Frobenius endomorphism came as a surprise to us since, over such rings $R$, there exist non-free modules $M$ such that $M \otimes_{R} M$ is torsion-free.
\end{abstract}

\section{Introduction}

Throughout the paper, $R$ denotes a commutative Noetherian ring and $\bmod R$ denotes the category of all finitely generated $R$-modules.

When $R$ is of prime characteristic $p$ and $M$ is an $R$-module, $\varphi^{n} M$ denotes the $R$-module obtained from $M$ by restriction of scalars along $\varphi^{n}$, where $\varphi: R \longrightarrow R$ is the Frobenius endomorphism given by $r \mapsto r^{p}$. Therefore the action of $R$ on $\varphi^{n} M$ is given by $r \cdot m=r^{p^{n}} m$ for $r \in R$ and $m \in M$. On the other hand, we agree that the $R$-module structure of the tensor product $M \otimes_{R} \varphi^{n} R$ comes from the right (ordinary) action of $R$ on $\varphi^{n} R$, i.e., $r \cdot(m \otimes s)=m \otimes(s r)$ for all $r, s \in R$ and $m \in M$. It follows that $M \otimes_{R} \varphi^{n} R \in \bmod R$ if $M \in \bmod R$.

When $(R, \mathfrak{m})$ is local of prime characteristic $p$, following [14], we set:

$$
\operatorname{drs}_{p}(R)=\inf \left\{p^{n} \mid \mathfrak{m}^{\left[p^{n}\right]} \subseteq(\underline{\mathbf{x}}) \text { for some system of parameters } \underline{\mathbf{x}} \text { of } R\right\},
$$

where $\mathfrak{m}^{\left[p^{n}\right]}$ of $\mathfrak{m}$ denotes the ideal generated by the $p^{n}$ th powers of any set of generators of $\mathfrak{m}$.

In this paper we are concerned with the following result of $\mathrm{Li}[13]$ :

Theorem 1.1 (Li $[13,2.4])$. Let $(R, \mathfrak{m})$ be a Cohen-Macaulay local ring of positive dimension and prime characteristic $p$ and let $M \in \bmod R$. Assume $n$ is an integer such that $p^{n} \geqslant \operatorname{drs}_{p}(R)$ and $M \otimes_{R} \varphi^{n} R$ is maximal Cohen-Macaulay. If $M$ has rank, then $M$ is free.

Sadeghi's research was supported by a grant from IPM.

Received June 28, 2017, revised October 17, 2017; published on February 21, 2018.

2010 Mathematics Subject Classification: 13D07, 13H10, 13A35.

Key words and phrases: Frobenius endomorphism, tensor product of modules, rank and torsion.

Article available at http://dx.doi.org/10.4310/HHA.2018.v20.n1.a15

Copyright (c) 2018, International Press. Permission to copy for private use granted. 
Recall that a module $M \in \bmod R$ has (constant) rank if there is a nonnegative integer $r$ such that $M_{\mathfrak{p}} \cong\left(R_{\mathfrak{p}}\right)^{\oplus r}$ for all associated prime ideals $\mathfrak{p}$ of $R$. Li $[13,2.5]$ points out that the following example from [14] shows that Theorem 1.1 could fail without the constant rank hypothesis.

Example 1.2 $([\mathbf{1 4}, 2.1 .7])$. Let $R=k \llbracket x, y \rrbracket /\left(x^{2}\right)$, where $k$ is a field of characteristic $p$, and let $M=R /(x y)$. Then $\operatorname{drs}_{p}(R)=p$ and $M \otimes_{R}{ }^{\varphi} R \cong R /\left(x^{p} y^{p}\right) \cong R$. Therefore $M \otimes_{R} \varphi R$ is maximal Cohen-Macaulay but $M$ is not free.

Our motivation comes from the fact that, in Example 1.2, for the minimal prime $\mathfrak{p}=(x) \in \operatorname{Spec}(R), M_{\mathfrak{p}}$ is not free over $R_{\mathfrak{p}}$. Hence $M$ is not locally free on the minimal prime ideals of $R$, which implies that $M$ does not have rank. Using an entirely different argument from [13], we are able to replace the rank hypothesis of Theorem 1.1 with the weaker condition that $M$ is locally free on the minimal primes of $R$, i.e., $M_{\mathfrak{p}}$ is free over $R_{\mathfrak{p}}$ for all minimal prime ideals $\mathfrak{p}$ of $R$. More precisely we prove:

Theorem 1.3. Let $(R, \mathfrak{m})$ be a Cohen-Macaulay local ring of positive dimension and prime characteristic $p$ and let $M \in \bmod R$. Assume that $n$ is an integer such that $p^{n} \geqslant \mathrm{e}(R)$ and $M \otimes_{R} \varphi^{n} R$ is maximal Cohen-Macaulay. If $M_{\mathfrak{p}}$ is free over $R_{\mathfrak{p}}$ for all minimal prime ideals $\mathfrak{p}$ of $R$, then $M$ is free.

Let us remark that the condition $M$ is locally free on the minimal prime ideals of $R$ holds, for example, when $R$ is reduced, even if $M$ does not have rank. Moreover, Example 1.2 shows that the hypothesis of $M$ being locally free on the minimal primes in Theorem 1.3 cannot be removed.

In the next section we give a proof of Theorem 1.3. As an application of our argument, we obtain the following result; see Corollary 2.6 for a more general statement.

Corollary 1.4. Let $R$ be a complete reduced non-regular Cohen-Macaulay local ring of prime characteristic $p$ that has a perfect residue field. Then for any $M \in \bmod R$, $\varphi^{n} M \otimes_{R} \varphi^{n} R$ is not maximal Cohen-Macaulay for all $n \gg 0$. In particular, $\varphi^{n} R \otimes_{R}$ $\varphi^{n} R$ is not maximal Cohen-Macaulay for all $n \gg 0$.

Prior to proceeding to our main argument, we discuss some results from the literature about tensor products of modules and compare them with Theorem 1.3 and Corollary 1.4.

Torsion properties of tensor products of modules over local rings were initially studied by Auslander [1], and Huneke and Wiegand [9]. Although tensor products tend to have torsion, it is not unnatural for a tensor product $M \otimes_{R} N$ to be torsionfree, or maximal Cohen-Macaulay, for some non-free modules $M$ and $N$. In fact, even when $M \otimes_{R} N$ is maximal Cohen-Macaulay, it does not force $M$ and $N$ to be torsionfree, or maximal Cohen-Macaulay, in general. For example, Huneke and Wiegand $[9,4.7]$ proved that, over one-dimensional non-Gorenstein domains, there always exist non-free modules $M$ such that $M \otimes_{R} M$ is maximal Cohen-Macaulay. On the other hand, over the domain $R=k \llbracket t^{3}, t^{4}, t^{5} \rrbracket$, there exists a module $M \in \bmod R$ which has torsion such that $M \otimes_{R} \omega$ is maximal Cohen-Macaulay, where $\omega$ is the canonical module of $R$; see [9, 4.8]. In the same direction, Constapel [6, 2.1] constructed modules $M$ and $N$ over the ring $R=k \llbracket t^{8}, t^{9}, t^{10}, t^{11}, t^{12}, t^{13} \rrbracket$, both of which have torsion, such that $M \otimes_{R} N$ is maximal Cohen-Macaulay. Let us also remark that whether or 
not there are such examples over complete intersection rings, mainly over those of codimension at least two, is an open question; see, for example, [5, 2.10].

In general, torsion properties of a tensor product $M \otimes_{R} N$ are significantly different when $M$ has rank, and when $M$ is locally free on the minimal primes. For example, Huneke and Wiegand [9,3.1] proved that, if $M \otimes_{R} N$ is maximal Cohen-Macaulay over a hypersurface ring $R$, then $M$ or $N$ is free (and hence both $M$ and $N$ are maximal Cohen-Macaulay) if and only if $M$ or $N$ has rank. This result easily fails when modules do not have rank: if $R=k \llbracket x, y \rrbracket /(x y)$ (where $k$ is any field, e.g., $k=\mathbb{F}_{p}$ ), $M=R /(x)$ and $N=R /\left(x^{2}\right)$, then $M \otimes_{R} M \cong M \otimes_{R} N \cong M$ is maximal Cohen-Macaulay; the modules $M$ and $N$ do not have rank but they are locally free on the minimal primes of $R$ (since $R$ is reduced). Theorem 1.3 and Corollary 1.4 show that, for all $n \gg 0$, there are no such examples for the case where $M=\varphi^{n} R$.

\section{Main result}

In this section we give a proof of our main result, Theorem 1.3. In preparation we prove two lemmas which seem to be of independent interest. First we recall:

2.1 ([2]). Let $R$ be a local ring and let $M \in \bmod R$ be a module. For a positive integer $i$, we denote by $\Omega^{i} M$ the $i$-th syzygy of $\mathrm{M}$, namely, the image of the $i$-th differential map in a minimal free resolution of $M$. As a convention, we set $\Omega^{0} M=M$.

The transpose $\operatorname{Tr} M$ of $M$ is defined as the cokernel of the $R$-dual map $\partial_{1}^{*}=$ $\operatorname{Hom}_{R}\left(\partial_{1}, R\right)$ of the first differential map $\partial_{1}$ in a minimal free resolution of $M$. Hence there is an exact sequence of the form $0 \rightarrow M^{*} \rightarrow P_{0}^{*} \rightarrow P_{1}^{*} \rightarrow \operatorname{Tr} M \rightarrow 0$. Note that the modules $\Omega^{i} M$ and $\operatorname{Tr} M$ are uniquely determined up to isomorphism, since so is a minimal free resolution of $M$, and there is a stable isomorphism $\Omega^{2} \operatorname{Tr} M \cong M^{*}$.

Lemma 2.2. Let $(R, \mathfrak{m})$ be a local ring and let $M, N \in \bmod R$. Assume that $n$ is a positive integer and that the following conditions hold:

(i) $M_{\mathfrak{p}}$ is free for all $\mathfrak{p} \in \operatorname{Spec} R-\{\mathfrak{m}\}$.

(ii) $\operatorname{depth}\left(M \otimes_{R} N\right) \geqslant n$.

(iii) $\operatorname{depth}(N) \geqslant n-1$.

Then $\operatorname{Ext}_{R}^{i}(\operatorname{Tr} M, N)=0$ for all $i=1, \ldots, n$.

Proof. Note that, if $r \geqslant 1$ and $\operatorname{Ext}_{R}^{r}(\operatorname{Tr} M, N) \neq 0$, then it follows from (i) that $\operatorname{Ext}_{R}^{r}(\operatorname{Tr} M, N)$ has finite length and hence $\operatorname{depth}\left(\operatorname{Ext}_{R}^{r}(\operatorname{Tr} M, N)\right)=0$.

We proceed by induction on $n$. Consider the following exact sequence from $[2,2.8]$ :

$$
0 \longrightarrow \operatorname{Ext}_{R}^{1}(\operatorname{Tr} M, N) \longrightarrow M \otimes_{R} N \longrightarrow \operatorname{Hom}_{R}\left(M^{*}, N\right) \longrightarrow \operatorname{Ext}_{R}^{2}(\operatorname{Tr} M, N) \longrightarrow 0 \text {. }
$$

Assume $n=1$. Suppose $\operatorname{Ext}_{R}^{1}(\operatorname{Tr} M, N) \neq 0$. It follows from (1) that $\operatorname{depth}\left(M \otimes_{R}\right.$ $N)=0$, which contradicts (ii). Therefore $\operatorname{Ext}_{R}^{1}(\operatorname{Tr} M, N)=0$.

Now assume $n=2$ and consider the following short exact sequence induced from (1):

$$
0 \longrightarrow M \otimes_{R} N \longrightarrow \operatorname{Hom}_{R}\left(M^{*}, N\right) \longrightarrow \operatorname{Ext}_{R}^{2}(\operatorname{Tr} M, N) \longrightarrow 0 .
$$

Suppose $\operatorname{Ext}_{R}^{2}(\operatorname{Tr} M, N) \neq 0$. $\operatorname{Since} \operatorname{depth}(N) \geqslant 1$, we have $\operatorname{depth}\left(\operatorname{Hom}_{R}\left(M^{*}, N\right)\right) \geqslant 1$; see, for example, $[4,1.2 .28]$. Hence the depth lemma and the exact sequence (2) imply that $\operatorname{depth}\left(M \otimes_{R} N\right)=1$, which contradicts (ii). Consequently, $\operatorname{Ext}_{R}^{2}(\operatorname{Tr} M, N)=0$. 
Now assume $n \geqslant 3$. Then the induction hypothesis yields $\operatorname{Ext}_{R}^{i}(\operatorname{Tr} M, N)=0$ for all $i=1, \ldots, n-1$. In particular, it follows from (1) that $M \otimes_{R} N \cong \operatorname{Hom}_{R}\left(M^{*}, N\right)$. So, by (ii), $\operatorname{depth}\left(\operatorname{Hom}_{R}\left(M^{*}, N\right)\right) \geqslant n$. Therefore it follows from [8, 2.3.3] that $\operatorname{Ext}_{R}^{i}\left(M^{*}, N\right)=0$ for all $i=1, \ldots, n-2$. Since $M^{*} \cong \Omega^{2} \operatorname{Tr} M$, we conclude that $\operatorname{Ext}_{R}^{i}(\operatorname{Tr} M, N)=0$ for all $i=1, \ldots, n$.

Our next result uses some techniques of Koh-Lee [11].

Lemma 2.3. Let $(R, \mathfrak{m}, k)$ be a Cohen-Macaulay local ring of positive dimension $d$ and prime characteristic $p$, and let $M \in \bmod R$. Assume that $n$ is an integer such that $p^{n} \geqslant \operatorname{drs}_{p}(R)$ and $p^{n} \geqslant \operatorname{drs}_{p}\left(R_{\mathfrak{p}}\right)$ for all minimal primes $\mathfrak{p}$ of $R$. If $t$ is a positive integer and $\operatorname{Ext}_{R}^{i}\left(M, \varphi^{n} R\right)=0$ for all $i=t, \ldots, t+d-1$, then $\operatorname{pd}(M)<t$. In particular, if $t=1$, i.e., if $\operatorname{Ext}_{R}^{i}\left(M, \varphi^{n} R\right)=0$ for all $i=1, \ldots, d$, then $M$ is free.

Proof. By [16, Theorem A], for every minimal prime ideal $\mathfrak{p}$ of $R$, we have $\operatorname{pd}_{R_{\mathfrak{p}}}\left(M_{\mathfrak{p}}\right)<\infty$, which then implies that $M_{\mathfrak{p}}$ is free over $R_{\mathfrak{p}}$.

To prove by contradiction, we assume $\operatorname{pd}(M) \geqslant t$. Consider a minimal free resolution of $M$ over $R$ :

$$
\mathbf{F}=\cdots \rightarrow F_{t+d} \stackrel{A_{t+d}}{\longrightarrow} F_{t+d-1} \stackrel{A_{t+d-1}}{\longrightarrow} \cdots \stackrel{A_{t+1}}{\longrightarrow} F_{t} \stackrel{A_{t}}{\longrightarrow} F_{t-1} \rightarrow \cdots .
$$

Then $F_{t} \neq 0$ and $A_{t} \neq 0$. Since $R$ is Cohen-Macaulay, all associated primes of $R$ are minimal. Thus there exists a minimal prime $\mathfrak{p}$ of $R$ such that $\left(A_{t}\right)_{\mathfrak{p}} \neq 0$, in which $\left(A_{t}\right)_{\mathfrak{p}}$ denotes the matrix over $R_{\mathfrak{p}}$ naturally derived from $A_{t}$ via localization at $\mathfrak{p}$. This localization process also gives rise to the following free resolution of $M_{\mathfrak{p}}$ over $R_{\mathfrak{p}}$ :

$\mathbf{F}_{\mathfrak{p}}=\cdots \rightarrow\left(F_{t+d}\right)_{\mathfrak{p}} \stackrel{\left(A_{t+d}\right)_{\mathfrak{p}}}{\longrightarrow}\left(F_{t+d-1}\right)_{\mathfrak{p}} \stackrel{\left(A_{t+d-1}\right)_{\mathfrak{p}}}{\longrightarrow} \cdots \stackrel{\left(A_{t+1}\right)_{\mathfrak{p}}}{\longrightarrow}\left(F_{t}\right)_{\mathfrak{p}} \stackrel{\left(A_{t}\right)_{\mathfrak{p}}}{\longrightarrow}\left(F_{t-1}\right)_{\mathfrak{p}} \rightarrow \cdots$.

As $M_{\mathfrak{p}}$ is free over $R_{\mathfrak{p}}$, the above resolution $\mathbf{F}_{\mathfrak{p}}$ is split exact. Thus the image of $\left(A_{t}\right)_{\mathfrak{p}}$ is a non-zero $R_{\mathfrak{p}}$-free direct summand of $\left(F_{t-1}\right)_{\mathfrak{p}}$. This further implies that $\varphi^{n}\left(\left(A_{t}\right)_{\mathfrak{p}}\right)$ is non-zero. (Indeed, applying the Frobenius functor to $\mathbf{F}_{\mathfrak{p}}$ affects neither its split exactness nor the ranks of the images of the differential maps.) In particular, $\varphi^{n}\left(A_{t}\right) \neq 0$. Hence $\varphi^{n}\left(A_{t}^{T}\right) \neq 0$, in which $A_{t}^{T}$ denotes the transpose of the matrix $A_{t}$.

Applying $\operatorname{Hom}_{R}\left(-, \varphi^{n} R\right)$ to $\mathbf{F}$ and using our assumption, we get the exact sequence:

$$
0 \leftarrow C \leftarrow F_{t+d} \stackrel{\varphi^{n}\left(A_{t+d}^{T}\right)}{\longleftarrow} F_{t+d-1} \stackrel{\varphi^{n}\left(A_{t+d-1}^{T}\right)}{\longleftarrow} \cdots \stackrel{\varphi^{n}\left(A_{t+1}^{T}\right)}{\longleftarrow} F_{t} \stackrel{\varphi^{n}\left(A_{t}^{T}\right)}{\longleftarrow} F_{t-1} \stackrel{B}{\longleftarrow} G
$$

in which $C$ is the cokernel of the map $\varphi^{n}\left(A_{t+d}^{T}\right)$ and $G$ is just a free $R$-module such that the above is a free resolution of $C$. Notice that all the entries of $\varphi^{n}\left(A_{i}^{T}\right)$ are in $\mathfrak{m}^{\left[p^{n}\right]}$. Moreover, by a property of free resolutions over a local ring, we see that, after a proper basis change, $B$ can be represented as $\left(\begin{array}{ll}I & 0 \\ 0 & B^{\prime}\end{array}\right)$ in block form, in which $I$ is an identity matrix and all the entries of $B^{\prime}$ are in $\mathfrak{m}$.

Next we claim that the row number of $B^{\prime}$ is positive. Indeed, if $B=\left(\begin{array}{ll}I & 0\end{array}\right)$ in block form, then the image of $B$ is $F_{t-1}$ and hence $\varphi^{n}\left(A_{t}^{T}\right)=0$, which is not the case.

As $p^{n} \geqslant \operatorname{drs}_{p}(R)$, there exists a system of parameters $\underline{\mathbf{x}}:=x_{1}, \ldots, x_{d}$ (hence a maximal $R$-regular sequence) such that $\mathfrak{m}^{\left[p^{n}\right]} \subseteq(\underline{\mathbf{x}})$. Let $E=E_{R /(\underline{\mathbf{x}})}(k) \cong$ $\operatorname{Hom}_{R}\left(R /(\underline{\mathbf{x}}), E_{R}(k)\right)$. It follows that $\operatorname{id}_{R}(E)=d=\operatorname{dim}(R)$ and $\mathfrak{m}^{\left[p^{n}\right]} E=0$. (Indeed, applying $\operatorname{Hom}_{R}\left(-, E_{R}(k)\right)$ to a minimal free resolution of $R /(\underline{\mathbf{x}})$ over $R$, we get a minimal injective resolution of $E$ over $R$.) 
Applying $\operatorname{Hom}_{R}(-, E)$ to the above resolution of $C$, we get

$$
0 \rightarrow E_{t+d} \stackrel{\varphi^{n}\left(A_{t+d}\right)}{\longrightarrow} \cdots \stackrel{\varphi^{n}\left(A_{t+1}\right)}{\longrightarrow} E_{t} \stackrel{\varphi^{n}\left(A_{t}\right)}{\longrightarrow} E_{t-1} \stackrel{B^{T}}{\longrightarrow} \operatorname{Hom}_{R}(G, E)
$$

in which both $E_{i}=\operatorname{Hom}_{R}\left(F_{i}, E\right)$ and $\operatorname{Hom}_{R}(G, E)$ are direct sums of $E$. Since $\operatorname{Ext}_{R}^{d+1}(C, E)$ vanishes, the above sequence must be exact at $E_{t-1}$. This is a contradiction, as the map $B^{T}$ is not injective (because of socle elements of $E_{t-1}$ and the presence of $\left(\begin{array}{c}0 \\ \left(B^{\prime}\right)^{T}\end{array}\right)$ as a non-trivial part of columns in $\left.B^{T}\right)$ while the map $\varphi^{n}\left(A_{t}\right)$ is 0 (because $\mathfrak{m}^{\left[p^{n}\right]} E_{t}=0$ ). (Alternatively, apply $-\otimes_{R} R /(\underline{\mathbf{x}})$ to the above resolution of $C$. The map $\varphi^{n}\left(A_{t}^{T}\right) \otimes 1_{R /(\underline{\mathbf{x}})}$ vanishes while the map $B \otimes 1_{R /(\underline{\mathbf{x}})}$ fails to be onto, which contradicts $\operatorname{Tor}_{d+1}^{R}(C, R /(\underline{\mathbf{x}}))=0$.)

Before giving our proof of Theorem 1.3, we need the following observation. Note that part of 2.4 has already been observed in the proof of $[7,2.2]$.

2.4. Let $(R, \mathfrak{m}, k)$ be a Cohen-Macaulay local ring with an infinite residue field $k$. Then, for all $\mathfrak{p} \in \operatorname{Spec} R$, it follows that

$$
\begin{aligned}
& \mathrm{e}(R) \geqslant \mathrm{e}\left(R_{\mathfrak{p}}\right)=\operatorname{length}_{R_{\mathfrak{p}}}\left(R_{\mathfrak{p}} /(\underline{\mathbf{x}}(\mathfrak{p}))\right) \quad \text { and } \\
& \left\lceil\log _{p}\left(\operatorname{length}_{R_{\mathfrak{p}}}\left(R_{\mathfrak{p}} /(\underline{\mathbf{x}}(\mathfrak{p}))\right)\right)\right\rceil \geqslant \log _{p}\left(\operatorname{drs}_{p}\left(R_{\mathfrak{p}}\right)\right),
\end{aligned}
$$

where $\mathrm{e}(R)$ is the multiplicity of $R, \underline{\mathbf{x}}(\mathfrak{p})$ denotes a minimal reduction of $\mathfrak{p} R_{\mathfrak{p}}$ in $\left(R_{\mathfrak{p}}, \mathfrak{p} R_{\mathfrak{p}}\right)$, and $\lceil-\rceil$ denotes the ceiling function. Note that such a reduction exists since $k(\mathfrak{p})$ is infinite for each $\mathfrak{p} \in \operatorname{Spec}(R)$.

In (3) the first inequality and the equality are well-known; see [12] and [4, 4.6.8], respectively (see also the proof of $[7,2.2]$ ). The second inequality is due to the fact that $\mathfrak{n}^{\text {length }(S / J)} \subseteq J$ for any $\mathfrak{n}$-primary ideal $J$ of a local ring $(S, \mathfrak{n})$.

We can now give our proof of Theorem 1.3. Recall that a local ring $R$ of prime characteristic $p$ is called $F$-finite if ${ }^{\varphi} R$, viewed as a module via the Frobenius endomorphism $\varphi$, is finitely generated; see, for example, [4, page 398].

A proof of Theorem 1.3. We can, if necessary, find a local ring extension $(S, \mathfrak{n})$ of $(R, \mathfrak{m})$ such that $S$ is F-finite, faithfully flat over $R$, with an infinite residue field, and $\mathfrak{m} S=\mathfrak{n}$. (For example, letting $\widehat{R} \cong k\left[\left[x_{1}, \ldots, x_{m}\right]\right] / I$ and using $\bar{k}$ to denote the algebraic closure of $k$, we can pick $S=\bar{k}\left[\left[x_{1}, \ldots, x_{m}\right]\right] / I \bar{k}\left[\left[x_{1}, \ldots, x_{m}\right]\right]$.) Then $S$ is a Cohen-Macaulay local ring, $\mathrm{e}(R)=\mathrm{e}(S)$, and $\operatorname{dim}(S)=\operatorname{dim}(R)$.

By going-down, all minimal prime ideals of $S$ contract to minimal primes of $R$. Thus $M \otimes_{R} S_{\mathfrak{p}}$ is free for all minimal prime ideals $\mathfrak{p}$ of $S$. Moreover,

$$
\left(M \otimes_{R} S\right) \otimes_{S} \varphi^{n} S \cong\left(M \otimes_{R} \varphi^{n} R\right) \otimes_{R} S
$$

is maximal Cohen-Macaulay over $S$; see [15, 23.3]. Note also that, $M \otimes_{R} S$ is free over $S$ if and only if $M$ is free over $R$. Therefore it suffices to prove the case where $R$ is F-finite and with an infinite residue field. By (3), we have $p^{n} \geqslant \operatorname{drs}_{p}\left(R_{\mathfrak{p}}\right)$ for all $\mathfrak{p} \in \operatorname{Spec}(R)$.

We now proceed by induction on $d=\operatorname{dim}(R)$. Let $d=1$ and let $M \otimes_{R} \varphi^{n} R$ be maximal Cohen-Macaulay. By Lemma 2.2, $\operatorname{Ext}_{R}^{1}\left(\operatorname{Tr} M, \varphi^{n} R\right)=0$ and so $\operatorname{Tr} M$ is free by Lemma 2.3. Hence $M$ is free. Now assume that $d>1$. By the induction hypothesis, 
$M_{\mathfrak{p}}$ is free for all non-maximal prime ideals $\mathfrak{p}$. Since $M \otimes_{R} \varphi^{n} R$ is a maximal CohenMacaulay $R$-module, by Lemma 2.2, we have:

$$
\operatorname{Ext}_{R}^{i}\left(\operatorname{Tr} M, \varphi^{n} R\right)=0 \text { for all } i=1, \ldots, d .
$$

Now the assertion follows from Lemma 2.3.

We finish this section with an application of Theorem 1.3 and give a proof of Corollary 1.4. We start by recording a few preliminary results, the first one being a special case of a result of Avramov, Hochster, Iyengar and Yao [3]: it is a strengthening of a classical result of Kunz [10] which considers the case where $M=R$.

$2.5([3,1.1])$. Let $R$ be a local ring of prime characteristic $p$ and let $0 \neq M \in \bmod R$. If $R$ is not regular, then $\operatorname{pd}_{R}\left(\varphi^{i} M\right)=\infty$ for all $i \geqslant 1$.

Let $n \geqslant 0$ be an integer. Recall that a module $M \in \bmod R$ satisfies Serre's condition $\left(S_{n}\right)$ if $\operatorname{depth}_{R_{\mathfrak{p}}}\left(M_{\mathfrak{p}}\right) \geqslant \min \left\{n, \operatorname{dim}\left(R_{\mathfrak{p}}\right)\right\}$ for all $\mathfrak{p} \in \operatorname{Supp}(M)$.

Note that every complete local ring of prime characteristic $p$ with a perfect residue field is F-finite; see [4, page 398]. Thus we reach a result that, in particular, establishes Corollary 1.4 advertised in the introduction.

Corollary 2.6. Let $(R, \mathfrak{m})$ be a reduced, F-finite Cohen-Macaulay local ring of positive dimension and prime characteristic $p$, and let $0 \neq M \in \bmod R$. Assume that $s, n$ and $t$ are positive integers such that $p^{n} \geqslant \mathrm{e}(R)$. If $\varphi^{s} M \otimes_{R} \varphi^{n} R$ satisfies Serre's condition $\left(S_{t}\right)$, then $R_{\mathfrak{q}}$ is regular for all $\mathfrak{q} \in \operatorname{Supp}(M)$ with $\operatorname{dim}\left(R_{\mathfrak{q}}\right) \leqslant t$. In particular, if $\varphi^{s} M \otimes_{R} \varphi^{n} R$ is maximal Cohen-Macaulay, then $R$ is regular.

Proof. Suppose that $\varphi^{s} M \otimes_{R} \varphi^{n} R$ satisfies Serre's condition $\left(S_{t}\right)$ and let $\mathfrak{q} \in \operatorname{Supp}(M)$ with $\operatorname{dim}\left(R_{\mathfrak{q}}\right) \leqslant t$. As $R_{\mathfrak{q}}$ is clearly regular when $\operatorname{dim}\left(R_{\mathfrak{q}}\right)=0$, we further assume $\operatorname{dim}\left(R_{\mathfrak{q}}\right)>0$. By $(3)$, we have that $p^{n} \geqslant \mathrm{e}\left(R_{\mathfrak{q}}\right)$. Moreover, since ${ }^{\varphi^{s}} M \otimes_{R} \varphi^{n} R$ satisfies Serre's condition $\left(S_{t}\right)$, it follows that ${ }^{\varphi^{s}} M_{\mathfrak{q}} \otimes_{R} \mathfrak{q}^{\varphi^{n}} R_{\mathfrak{q}}$ is a maximal Cohen-Macaulay $R_{\mathfrak{q}}$-module. Hence we conclude from Theorem 1.3 that ${ }^{\varphi^{s}} M_{\mathfrak{q}}$ is free over $R_{\mathfrak{q}}$. So the conclusion follows from 2.5.

\section{Acknowledgments}

We are grateful to Tokuji Araya for his feedback on the manuscript.

\section{References}

[1] Maurice Auslander, Modules over unramified regular local rings, Illinois J. Math. 5 (1961), 631-647.

[2] Maurice Auslander and Mark Bridger, Stable Module Theory, Mem. Amer. Math. Soc., vol. 94, Amer. Math. Soc., Providence, 1969.

[3] Luchezar L. Avramov, Melvin Hochster, Srikanth B. Iyengar, and Yongwei Yao, Homological invariants of modules over contracting endomorphisms, Math. Ann. 353(2) (2012), 275-291.

[4] Winfried Bruns and Jürgen Herzog, Cohen-Macaulay Rings, Cambridge Stud. Adv. Math., vol. 39, Cambridge University Press, Cambridge, 1993. 
[5] Olgur Celikbas and Roger Wiegand, Vanishing of Tor, and why we care about it, J. Pure Appl. Algebra 219(3) (2015), 429-448.

[6] Petra Constapel, Vanishing of Tor and torsion in tensor products, Comm. Algebra 24 (1996), 833-846.

[7] Douglas J. Dailey, Srikanth B. Iyengar, and Thomas Marley, Detecting finite flat dimension of modules via iterates of the Frobenius endomorphism, to appear in J. Commut. Algebra, https://projecteuclid.org/euclid.jca/ 1496541671.

[8] Hailong Dao, Some homological properties of modules over a complete intersection, with applications, Commutative Algebra, pages 335-371, Springer, New York, 2013.

[9] Craig Huneke and Roger Wiegand, Tensor products of modules and the rigidity of Tor, Math. Ann. 299(3) (1994), 449-476.

[10] Ernest Kunz, Characterizations of regular local rings for characteristic $p$, Amer. J. Math. 91 (1969), 772-784.

[11] Jee H. Koh and Kisuk Lee, Some restrictions on the maps in minimal resolutions, J. Algebra 202 (1998), 671-689.

[12] Christer Lech, Inequalities related to certain couples of local rings, Acta Math. 112 (1964), 69-89.

[13] Jinjia Li, Frobenius criteria of freeness and Gorensteinness, Arch. Math. 98 (2012), 499-506.

[14] Claudia Miller, The Frobenius endomorphism and homological dimensions, Commutative Algebra (Grenoble/Lyon, 2001), Contemp. Math., vol. 331, pages 207-234, Amer. Math. Soc., Providence, RI, 2003.

[15] Hideyuki Matsumura, Commutative Ring Theory, Cambridge Stud. Adv. Math., vol. 8, Cambridge University Press, Cambridge, 1989, xiv+320 pp., translated from the Japanese by M. Reid, second edition.

[16] Saeed Nasseh, Massoud Tousi, and Siamak Yassemi, Characterization of modules of finite projective dimension via Frobenius functors, Manuscripta Math. 130 (2009), 425-431.

Olgur Celikbas olgur.celikbas@math.wvu.edu

Department of Mathematics, West Virginia University, Morgantown, WV 26506-6310, U.S.A.

Arash Sadeghi sadeghiarash61@gmail.com

School of Mathematics, Institute for Research in Fundamental Sciences (IPM), P.O. Box 19395-5746, Tehran, Iran

Yongwei Yao yyao@gsu.edu

Department of Mathematics and Statistics, Georgia State University, Atlanta, GA 30303, U.S.A. 